\title{
La comunicación entre padres e hijos adolescentes y el uso del celular: un estudio comparativo en zona rural y urbana
}

\section{Communication between parents and adolescent' children, and mobile phone use: a comparative study in rural and urban areas}

\section{Diana Marcela Rodríguez-Clavijo ${ }^{1}$ Leidy Camila Guzmán-Patiño? Diana Ximena Puerta-Cortés ${ }^{3}$}

(2)

Recepción: 15/09/2020
-

Aprobación: 14/10/2020
-

Publicación: 18/12/2020

\section{Para citar este artículo:}

Rodríguez-Clavijo, D. M., Guzmán-Patiño, L. C., \& Puerta-Cortés, D. X. (2020).

La comunicación entre padres e hijos adolescentes y el uso del celular: un estudio comparativo en zona rural y urbana. Indagare, (8), 30-38.

https://doi.org/10.35707/indagare/803

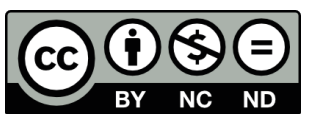

\footnotetext{
${ }^{1}$ Grupo de investigación GESS, Universidad de Ibagué, Colombia. ORCID: 0000-0002-2068-1742. Correo electrónico: 3220161049@unibague.edu.co

${ }^{2}$ Grupo de investigación GESS, Universidad de Ibagué, Colombia. ORCID: 0000-0001-5650-4521. Correo electrónico: 3220161082@unibague.edu.co

${ }^{3}$ Grupo de investigación GESS, Universidad de Ibagué, Colombia. ORCID: 0000-0003-4134-2421. Correo electrónico: diana.puerta@unibague.edu.co
} 


\title{
Resumen
}

El objetivo del estudio fue analizar las diferencias entre la comunicación de padres e hijos adolescentes y el uso problemático del celular en una zona rural y urbana del departamento del Tolima. En el estudio exploratorio con diseño transversal, participaron 300 adolescentes entre los 13 y 18 años, a quienes se les aplicó el cuestionario de datos sociodemográficos, la escala de comunicación padres e hijos Parent-Adolescent Communication Scale (PACS) y la escala de uso problemático del celular Mobile Phone Problem Use Scale (MPPUSA). Los resultados muestran asociación entre la percepción de la calidad en la comunicación con la madre y la zona de residencia. Respecto al uso del celular, preadolescentes y adolescentes hacen uso diferencial, aunque no se evidenció asociación entre el grado de uso del celular y el lugar donde habitan. En conclusión, percibir una comunicación excelente con los padres no es determinante del tipo de uso del celular (moderado o problemático), es posible que las percepciones de los hijos sobre los estilos parentales influyan en el uso de la tecnología. Aun así, se requiere de más investigación para soportar los hallazgos.

\begin{abstract}
The aim of this study was to analyze the differences between the communication of parents and adolescent children, and the problematic mobile phone use in rural and urban areas of the department of Tolima. In the exploratory study with a cross-sectional design, 300 adolescents between 13 and 18 years old participated, to whom the sociodemographic data questionnaire, the Parent-Adolescent Communication Scale (PACS) and Mobile Phone Problem Use Scale (MPPUSA), was applied. The results show an association between the perception of quality in communication with the mother and the area of residence. Regarding mobile phone use, pre-adolescents and adolescents make differential use; although no association was evidenced between the degree of mobile phone use and the place where they live. In conclusion, perceiving excellent communication with parents is not a determining factor of the type of mobile phone use (moderate or problematic), it is possible that children's perceptions of parenting styles influence the use of technology. Even so, more research is required to support the findings.
\end{abstract}

Palabras claves: Comunicación, uso del celular, rural, urbano,
adolescentes.
Key words: Communication, mobile phone use, rural, urban, adolescents. 


\section{Introducción}

La comunicación es un proceso indispensable para el intercambio de ideas, creencias y valores que son útiles para tomar decisiones en cualquier contexto (Fernández, McFarlane, González, Díaz, Betancourt-Díaz, Cintrón-Bou \& Villaruel, 2017). Al ser la relación familiar entre padres e hijos la más temprana y duradera de todas las relaciones sociales, influye en las aptitudes y en la capacidad de afrontamiento ante las situaciones que enfrenten los hijos (Guaita, 2010). Entonces, una adecuada comunicación en el entorno familiar desde la niñez hace que los hijos adquieran la capacidad para establecer relaciones con los demás, desarrollar la empatía y, lo más importante, comunicar a los otros aquello que sienten; de esta manera, se cumple con la función adaptativa de la comunicación ante el entorno (Crespo, 2011; Saarni, 1999). En cambio, la comunicación deficiente se caracteriza por contextos familiares de control y autoritarismo o lo contrario, evadir la responsabilidad y dejar a los docentes el compromiso de la formación y educación de sus hijos, no solo académicamente, sino también en aspectos personales (Guaita, 2010).

La adolescencia es la etapa de cambios fisiológicos, cognitivos, morales, sociales y de personalidad (Gaete, 2015). Esta etapa va desde los 11 a los 20 años, en ella la persona se encuentra influenciada por la dinámica familiar, la experiencia escolar, el marco cultural y social, las condiciones económicas y políticas del momento. Por lo anterior, la construcción de una identidad propia puede llegar a significar un rechazo al mundo de los adultos y surgir un distanciamiento con sus padres lo cual genera poca comunicación con ellos, dado que a los chicos no les interesa hablar con sus padres, y estos a la vez insisten en conocerlo todo, ocasionando enfrentamientos y choques (De la Villa Moral \& Suárez, 2016).

Con la aparición de las Tecnologías de la Información y la Comunicación (TIC), surgieron cambios significativos en la sociedad relacionados con el uso de la información y el establecimiento de las relaciones al interior de las familias (López-Fernández, Honrubia-Serrano \& Freixa-Blanxart, 2012). En la actualidad, debido al fácil acceso que tienen los adolescentes a las TIC, se presentan cambios positivos o negativos en su forma de expresión, en el establecimiento de hábitos, actitudes y en sus relaciones con el entorno (López-Fernández et al., 2012).

Se ha encontrado que son los adolescentes quienes tienen mayor uso del celular. Cerca de 279 a 528 minutos por día, el $92 \%$ se conecta en el celular todos los días y el $24 \%$ lo utiliza en reiteradas ocasiones (Lenhart et al., 2015). Lo anterior se debe a que el celular es un elemento electrónico novedoso, atractivo y accesible, que incorpora funciones similares a las de una computadora; por ejemplo, el almacenamiento de datos, la navegación, el correo electrónico y la instalación de software (Kuntsche \& Labhart, 2014). 
No obstante, cuando el adolescente desarrolla conductas repetitivas con el celular (consultar redes sociales, jugar, navegar) y es incapaz de regular el tiempo de uso, se generan consecuencias conductuales, sociales y afectivas en su vida, hasta el punto de iniciar síntomas de dependencia; fenómeno denominado como uso problemático del celular (Pedrero, Rodríguez \& Ruiz, 2012). Se presentan comportamientos potencialmente dañinos y perturbadores, que interfieren en espacios fundamentales de las personas, como la familia, el estudio y el trabajo, que se han asociado con la adicción. Sin embargo, aún la literatura no reporta la existencia de la adicción al celular, por tanto, se empleará el término uso problemático para efectos de este texto (Billieux, Maurage, López-Fernández, Kuss \& Griffiths, 2015).

En 2017, en el departamento del Tolima, se entregó en la ciudad de Ibagué la primera zona wifi gratis, 8700 tabletas para educar, el octavo Punto Vive Digital Lab del país y 58 Kioscos Vive Digital. En ese mismo año la cobertura de internet se fue ampliando a las zonas rurales y semirurales, como por ejemplo el municipio de Rovira, que evidencia un aumento en la penetración de internet en Colombia (MinTIC, 2017). En consecuencia, el libre y fácil acceso a internet a través de diferentes plataformas puede generar conductas desadaptativas en los más jóvenes, como es el uso problemático del celular que es un fenómeno cada vez más presente en la vida de los adolescentes (Billieux et al., 2015). No obstante, la mayoría de estudios relacionados con esta problemática se han efectuado en los cascos urbanos (De la Villa Moral \& Suárez, 2016; Lepp, Barkley \& Karpinski, 2015; Tresáncoras, García-Oliva \& Piqueras, 2017; Yudes-Gómez, BaridonChauvie \& Gonzáles-Cabrera, 2018).

Según lo anterior, se requiere realizar estudios que permitan comprender la incidencia del uso del celular en el contexto colombiano, se cuenta con poca evidencia empírica acerca del impacto de las nuevas tecnologías en la comunicación familiar, en especial en zonas rurales. Por tanto, el objetivo del estudio fue analizar las diferencias entre la comunicación de padres e hijos adolescentes y el uso problemático del celular, en una muestra de estudiantes de zona rural y urbana del departamento del Tolima.

\section{Materiales y métodos}

Estudio exploratorio con diseño trasversal (Hernández, Fernández \& Baptista, 2010). Con una muestra de tipo no probabilística intencional conformada por 300 participantes, de ellos 150 provenían de la zona rural (ver Figura 1) y 150 de la zona urbana. Del total de la muestra, el $50 \%$ eran mujeres, el 52,3 \% preadolescentes y el 47,7 \% adolescentes. El promedio de edad fue 15,5 años (DT=1.1), con un rango de edad de 13 a 18 años. 
Figura 1. Institución Educativa Técnica La Ceiba en Rovira (Tolima)

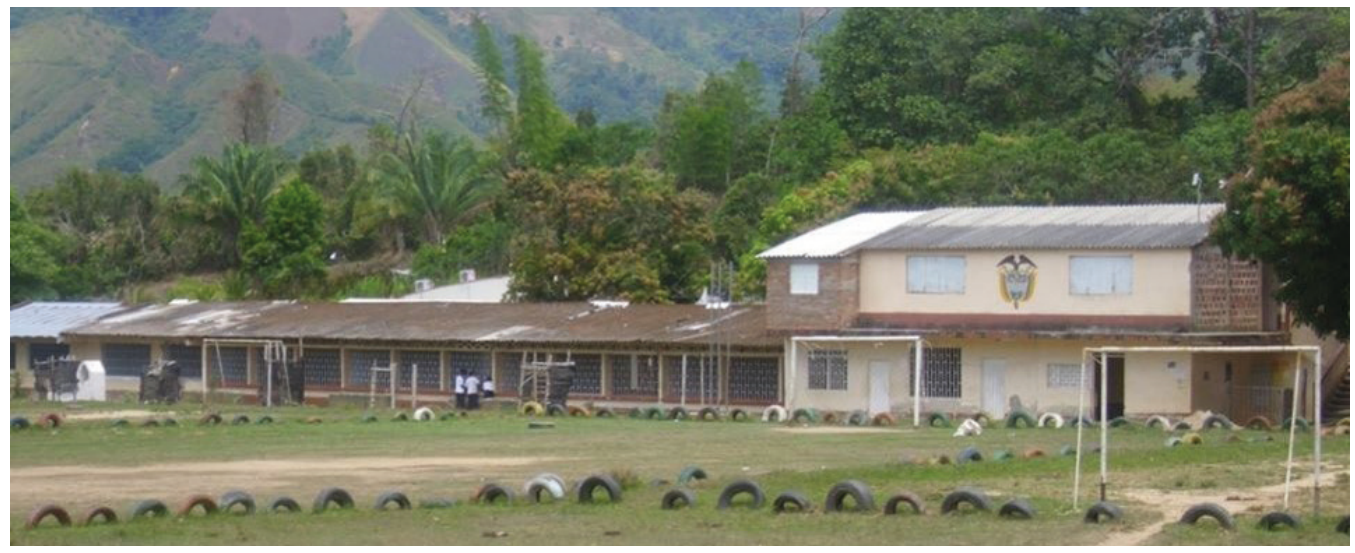

Fuente: Sandra Liliana Patiño

Para recolectar los datos se aplicó el cuestionario de datos sociodemográficos, la escala comunicación padres e hijos PACS (Barnes \& Olson, 1982-1985), con el fin de identificar la existencia de problemas en la comunicación entre padres e hijos y la escala uso problemático del celular MPPUSA (Bianchi \& Phillips, 2005), en su adaptación a la lengua española realizada por López-Fernández, Honrubia-Serrano \& Freixa-Blanxart (2012). Los participantes/padres o tutores diligenciaron el consentimiento informado y luego los estudiantes completaron los cuestionarios de manera voluntaria (ver Figura 2). Posterior a la aplicación se analizaron los resultados a través del Software Statistical Package for the Social Sciences (SPSS) versión 24.0.

Figura 2. Sesión de aplicación de los cuestionarios en Rovira (Tolima)

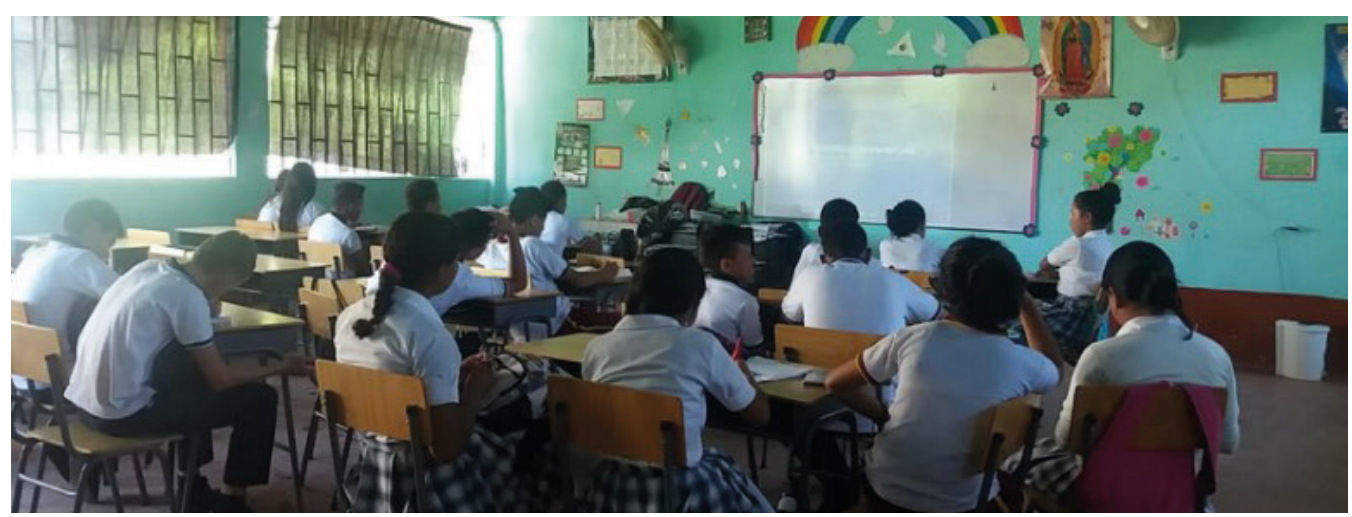

Fuente: Sandra Liliana Patiño 


\section{Resultados}

Los resultados muestran asociación entre la percepción de la calidad en la comunicación con la madre y la zona. De ellas, el 31,3 \% de la zona urbana reportó una excelente comunicación, el 27,7 \% comunicación aceptable y el 16,7 \% deficiente. En la zona rural, el 38,8 \% manifestó tener comunicación deficiente, el 30,3\% regular y el 17,3\% excelente $\left(\chi^{2}=24,51 ; p=0.00\right)$. Respecto a la calidad de la comunicación con el padre y la zona, no se encontraron diferencias significativas $\left(\chi^{2}=4,74 ; p=0.00\right)$. Estos datos se deben, posiblemente, a que en la zona urbana se interactúa con mayor número de actividades que le permiten a la madre establecer mejores relaciones con su mismo género (OsorioToloza, 2015).

Frente al uso del celular y la zona, se encontró que el $26 \%$ de la rural y el $20 \%$ de la urbana presentaban uso problemático del celular; el $50 \%$ de la urbana y el 38,7 \% de la rural puntuaron en uso en riesgo del celular; mientras el $30 \%$ de la rural y el 35,3\% de la urbana presentaban uso moderado del celular. A pesar de estos resultados no se evidenció asociación significativa entre el grado de uso del celular y el lugar donde habitan $\left(\chi^{2}=4,00\right.$; $p=0.13$ ), debido, posiblemente, a que durante los últimos años las nuevas tecnologías se han extendido por igual en zonas rurales y urbanas. Esto se debe a los esfuerzos del MinTIC por llevar conectividad alrededor del territorio colombiano, con el fin de reducir la brecha digital en las diferentes regiones del país (MinTIC, 2017; MinTIC, 2020).

No obstante, al comparar el grado de uso del celular y la etapa del desarrollo, se evidencia asociación entre las variables (ver Tabla 1).

Tabla 1. Grado de uso del celular y etapa del desarrollo

\begin{tabular}{|c|c|c|c|c|c|c|}
\hline \multirow{3}{*}{ Etapa } & \multicolumn{3}{|c|}{ Uso del celular } & \multirow{3}{*}{ Total } & \multirow{3}{*}{$\chi^{2}$} & \multirow{3}{*}{$p$} \\
\hline & Moderado & En riesgo & Problemático & & & \\
\hline & $\%(\mathrm{n})$ & $\%(\mathrm{n})$ & $\%(\mathrm{n})$ & & & \\
\hline Preadolescente & $39,5(62)$ & $42,7(67)$ & $17,8(28)$ & 157 & & \\
\hline Adolescente & $25,2(36)$ & $46,2(66)$ & $28,7(41)$ & 143 & 8,72 & 0,013 \\
\hline
\end{tabular}

Fuente: elaboración propia 
Los resultados de la Tabla 1 evidencian que el uso del celular es diferencial entre preadolescentes y adolescentes, parece que la adolescencia es el periodo más sensible en el uso de tecnologías. Los adolescentes de la muestra del estudio presentan uso problemático del celular, mientras que los preadolescentes uso moderado del celular. Es posible que en la adolescencia la necesidad social de estar en contacto constante con su grupo de iguales, en aras de establecer su identidad a través de la pertenencia a grupos, incremente el uso del celular (Lepp, Barkley \& Li, 2017).

En relación con el grado escolar y al grado de uso del celular, no se evidenció asociación $\left(\chi^{2}=2,21 ; p=0.69\right)$. No obstante, se demuestra asociación entre la percepción de la calidad de la comunicación con el padre y el grado de uso del celular ( $\chi 2=14,15$; $p=0.02$ ). El $37,7 \%$ de la muestra presenta uso problemático del celular y percibe una excelente calidad en la comunicación con su padre, el $62,2 \%$ presenta uso moderado y una comunicación deficiente o regular con el padre y el 43,2 \% con uso problemático del celular percibe una comunicación regular o deficiente con su padre. Además, el análisis de relaciones arroja una correlación significativa positiva y débil, entre los puntajes del MMPPUSA y los puntajes totales del PACS (madre) Rho $=0,12 ; \mathrm{p}=0,01$ y PACS (padre) Rho $=0,14 ; p=0,01$.

Según lo anterior, percibir una comunicación excelente con los padres no es determinante del tipo de uso del celular (moderado, problemático). Es posible que los estilos parentales (democrático, autoritario, negligente, indulgente) y la percepción de los hijos sobre estos estilos influya en sus decisiones en el uso de la tecnología. Es probable que un estilo indulgente en el que el padre es responsable pero no exigente, el hijo tenga menos supervisión por el uso del celular y, aun así, perciba una adecuada comunicación con sus padres (Capano Bosh, González Tornaría \& Massonnier, 2016). A pesar de ello se requiere de más investigación que permita ampliar estos hallazgos.

\section{Potencial uso}

Los datos de este estudio permitieron identificar la calidad de la comunicación entre los padres e hijos y el uso del celular en una muestra de zona rural y urbana del departamento del Tolima. Esta información permitió identificar que la comunicación con la madre es diferencial en las dos zonas, hallazgo que promueve el desarrollo de intervenciones para el mejoramiento de la comunicación al interior de las familias y el fortalecimiento de los vínculos con los hijos, en especial, en los sectores rurales. Además, en las dos zonas se debe incentivar el manejo apropiado del celular en los adolescentes, con el fin de prevenir las distintas problemáticas que surgen por el uso inadecuado y a edades tempranas. 
INDAGA]EE e-ISSN: 2357-5042 • Número 8 (2020) • Universidad de Ibagué • doi: https://doi.org/10.35707/indagare/803

Ficha técnica del proyecto

Título del proyecto: Policonsumo de drogas y adicciones tecnológicas: caracterización en muestras de jóvenes colombianos y paraguayos.

PRIT: Educación y bienestar para el desarrollo humano integral.

Código del proyecto: 19-496-INT.

Palabras claves: Policonsumo, drogas, adicciones tecnológicas, colombianos, paraguayos, sustancias psicoactivas.

Grupo de investigación: Educación, Salud y Sociedad, GESS.

Investigadora principal: Diana Ximena Puerta-Cortés.

Correo electrónico: diana.puerta@unibague.edu.co

\section{Referencias}

Billieux, J., Maurage, P., Lopez-Fernandez, O., Kuss, D. J., \& Griffiths, M. D. (2015). Can disordered mobile phone use be considered a behavioral addiction? An update on current evidence and a comprehensive model for future research. Current Addiction Reports, 2(2), 156-162. https://doi.org/10.1007/s40429015-0054-y

Capano, A., González, M. L., \& Massonnier, N. (2016). Estilos relacionales parentales: estudio con adolescentes y sus padres. Revista de Psicología, 34(2), 413-444. http://dx.doi.org/10.18800/ psico.201602.008

Crespo, J. (2011). Bases para construir una comunicación positiva en la familia. Revista de Investigación en Educación, 9(2), 91-98. Recuperado de http://reined.webs.uvigo.es/index.php/reined/article/ view/116

De la Villa Moral, M., \& Suárez, C. (2016). Factores de riesgo en el uso problemático de Internet y del teléfono móvil en adolescentes españoles. Revista Iberoamericana de Psicología y Salud, 7(2), 69-78. https://doi.org/10.1016/j.rips.2016.03.001

Fernández, A. M., McFarlane, M. N., González, R., Díaz, L., Betancourt-Díaz, E., Cintrón-Bou, F., \& Villarruel, A. (2017). Actitudes hacia la comunicación sexual entre padres/madres y adolescentes en Puerto Rico. Revista puertorriqueña de psicología, 28(1), 80-95. Recuperado de https://www.ncbi. nlm.nih.gov/pmc/articles/PMC5520656/

Gaete, V. (2015). Desarrollo psicosocial del adolescente. Revista Chilena de Pediatría, 86(6), 436-443. https://doi.org/10.1016/j.rchipe.2015.07.005

Guaita, V. (2010). La evaluación de la percepción de la comunicación emocional de los padres en niños desde la neuropsicología. Interdisciplinaria, 27(1), 129-146. Recuperado de https://www.redalyc.org/ pdf/180/18014748009.pdf

Hernández, R., Fernández, C., \& Baptista, L P. (2010). Metodología de la investigación. México: McGrawHill. 
INDAGAZE e-ISSN: 2357-5042 • Número 8 (2020) • Universidad de Ibagué • doi: https://doi.org/10.35707/indagare/803

Kuntsche, E., \& Labhart, F. (2014). (2014). The future is now-using personal cellphones to gather data on substance use and related factors. Addiction, 109(7), 1052-1053. https://doi.org/10.1111/add.12540

Lenhart A., Duggan M., Perrin A., Stepler R., Rainie H., \& Parker K. (2015). Teens, social media and technology overview. Smartphones facilitate shifts in communication landscape for teens. Washington, D. C.: Pew Internet \& American Life Project.

Lepp, A., Barkley, J. E., \& Karpinski, A. C. (2015). The relationship between cell phone use and academic performance in a sample of US college students. Sage Open, 5(1), 1-9. https://journals.sagepub.com/ doi/full/10.1177/2158244015573169

Lepp, A., Barkley, J. E., \& Li, J. (2017). Motivations and experiential outcomes associated with leisure time cell phone use: Results from two independent studies. Leisure Sciences, 39(2), 144-162. https://doi.org/10.1080/01490400.2016.1160807

López-Fernández, O., Honrubia-Serrano, M. L., \& Freixa-Blanxart, M. (2012). Adaptación española del "Mobile Phone Problem Use Scale" para población adolescente. Adicciones, 24(2), 123-130. Recuperado de http://www.adicciones.es/index.php/adicciones/article/view/104

MinTIC. (2017). El Tolima se fortalece en tecnología con entrega de zona wifi gratis y Kioscos Vive digital. Recuperado de https://n9.cl/vk7a8

MinTIC. (2020). Las TIC siguen impactando positivamente la educación colombiana. Recuperado de https://n9.cl/6b3um

Osorio-Toloza, K. (2015). Calidad de vida de adolescentes de zonas urbana y rural: comparación de la calidad de vida de adolescentes de zonas urbana y rural de la Provincia de García Rovira, Santander (tesis de pregrado). Universidad Santo Tomas, Bogotá, Colombia.

Pedrero, E., Rodríguez, M., \& Ruiz, J. (2012). Adicción o abuso del teléfono móvil. Revisión de la literatura. Adicciones, 24(2), 139-152. Recuperado de http://www.adicciones.es/index.php/adicciones/article/ view/107

Saarni, C. (1999). The development of emotional competence. New York: The Guilford Press.

Tresáncoras, A. G., García-Oliva, C., \& Piqueras, J. A. (2017). Relación del uso problemático de WhatsApp con la ansiedad y personalidad en adolescentes. Health and Addictions. Salud y drogas, 17(1), 27-36. https://doi.org/10.21134/haaj.v17i1.272

Yudes-Gómez, C., Baridon-Chauvie, D., \& González-Cabrera, J. (2018). Ciberacoso y uso problemático de internet en Colombia, Uruguay y España: un estudio transcultural. Comunicar, 26(56), 49-58. https://doi.org/10.3916/C56-2018-05 